\title{
Determination of Sewer Structure Using a Moving Camera
}

\author{
S.Fu' ${ }^{\mathrm{P}}$ D. Cooper ${ }^{\mathrm{P}}$, T.Pridmore ${ }^{\mathrm{P}}$ and N. Taylor ${ }^{\mathrm{F}}$ \\ P School of Engineering IT, Sheffield Hallam University, City Campus, Sheffield, S1 \\ 1WB, United Kingdom \\ School of Construction, Sheffield Hallam University, City Campus, Sheffield, S1 \\ 1WB, United Kingdom
}

\begin{abstract}
This paper presents a novel method of obtaining an accurate estimate of the shape of a sewer from a sequence of images captured by a camera moving along its length. An image flow technique is first applied. This gathers information from the whole image plane to produce a global description of the changes in image feature positions due to camera movement. Given this description, the motion of the camera may be estimated. The motion parameters obtained are then exploited by a structure-from-motion technique which estimates the shape of the sewer pipe by considering the motion of individual image features. Sewer shape data is managed by a specially developed Graphical User Interface which allows the user to view the shape of the sewer section from different view angles.
\end{abstract}

\section{Introduction}

Over the last decade, the main technique used in sewer survey has been to consider the image sequence recorded by a CCTV (Closed Circuit Television) camera mounted on a sled moving along the sewer. On the basis of this image sequence a human operator performs a broad classification of each section of the sewer. The qualitative description produced is sufficient to identify the portions that are in urgent need of attention but not to identify the fractional sewer deformation which could be used to monitor structural changes in the sewer system. Such deformation could only be detected by a quantitative analysis of the recorded image sequences.[1]

This paper presents a novel method of obtaining an accurate estimate of the shape of a sewer from a sequence of images captured by a camera moving along its length. Here, shape is defined as the $3 \mathrm{D}$ position of a set of feature points lying on the sewer wall. Generally speaking, if the motion of the camera, or, more specifically, the geometrical relationship between adjacent viewpoints, is known, the 3D shape of the object under investigation can be computed from the images taken from those viewpoints. Equally, if the 3D structure of the object is known, the camera motion can be obtained. However, in the sewer application, although the original profile of the pipe is available, neither deformed shape nor the camera motion is known precisely. 
In the method presented, an image flow [2][3] technique is first applied. This gathers information from the whole image to produce a global description of the changes in image feature positions due to camera movement. Given this description, the motion of the camera may be estimated. The motion parameters obtained are then exploited by a structure-from-motion [4] technique which estimates the shape of the sewer pipe by considering the motion of individual image features. Any image features may be used, the only requirement being that they reflect stable features of the sewer wall.

The current paper presents an analysis of the measurement error expected from the proposed technique and demonstrates the application of the technique to small-bore sewer survey. The image sequence used was acquired from a VHS videotape record of a commercially performed survey. In the circumstances considered, the expected measurement error is not more than $0.4 \%$ of the measurement distance. Also demonstrated is a Graphical User Interface (GUI) which allows the user to view the shape of the sewer section from different view angles.

\section{Camera Motion and 3-D Structure Recovery}

Triangulation can produce three dimensional measurement of spatial points. The accuracy of the measurement mainly depends on the precision with which the geometrical relationship between two viewpoints is known. The images obtained as a camera moves observing an object could be considered to be a series of images from different viewpoints. Study of the camera motion is then required in order to obtain the precise geometrical relationship between adjacent viewpoints.

Because of the tough travelling conditions inside a sewer, the camera motion cannot controlled precisely. In order to recover the exact camera motion, its relationship to the image sequence needs to be studied.

The sewer can be assumed to be locally straight, so that during the period when a short image sequence is taken (it takes about 0.1 second to capture 3 images), the camera can be assumed to be moving in a straight line. Fig. 1 shows the camera at three successive positions.

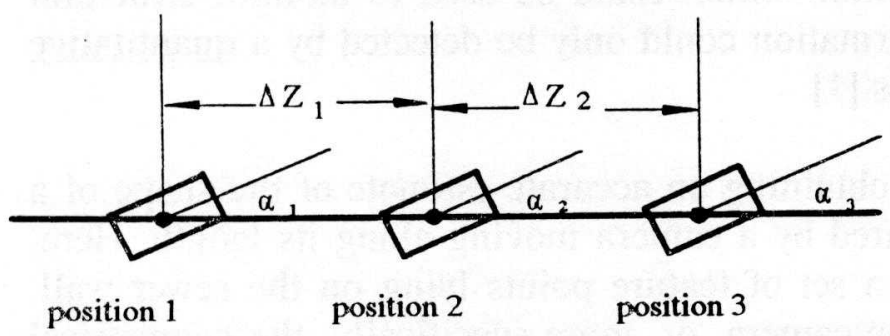

Fig. 1 Illustration of a camera moving forward
The angle between the camera's optical axis and the direction of camera motion may change during the procedure. This angle is termed the observing-moving angle (OMA) in this paper. These angles are indicated as $\alpha_{1}, \alpha_{2}$ and $\alpha_{3}$ at positions 1,2 and 3 in Fig. 1 . A distance $\Delta Z_{1}$ separates position 1 and 2 while $\Delta Z_{2}$ separates position 2 and 3. 
In order to relate the camera motion to the images taken, two co-ordinate systems are used. This is illustrated in Fig.2. One co-ordinate system is the world system $(O X Y Z)$ while the other is the camera system $\left(O X_{1} Y Z_{1}\right)$. The origin of both systems is at the centre of the camera lens. The $\mathrm{OZ}$ axis is the direction in which the camera is moving at point $O$ while $O Z_{1}$ is coincident with the optical axis of the camera. The image plane of the camera, $x O_{1} y$, is perpendicular to $O Z_{1}$, with $O O_{1}$ the focal length $f$ of the camera lens. The OMA, the intersection angle between

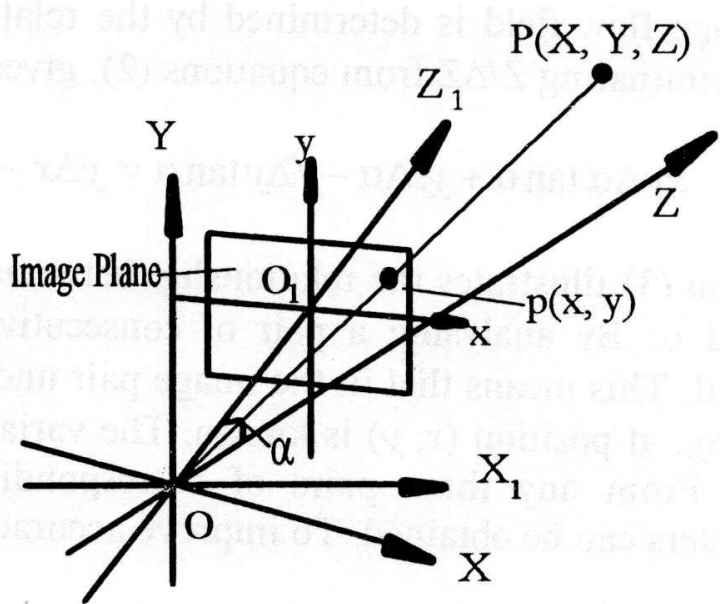

Fig. 2 The Combined Co-ordinate System $O Z$ and $O Z_{1}$, is $\alpha$. Note that the $O Y$ axis is not necessarily vertical; it is perpendicular to the plane defined by $O Z$ and $O Z_{1}$.

Suppose that an arbitrary fixed point is located at $(X, Y, Z)$ in world co-ordinates, and was observed by the camera with its image, $p$, located at $(x, y)$ on the image plane. The relationship between $P$ and $p$ is

$$
\left\{\begin{array}{l}
x=f \cdot \frac{X \cos a+Z \sin a}{Z \cos a-X \sin a} \\
y=f \cdot \frac{Y}{Z \cos a-X \sin a}
\end{array}\right.
$$

As the camera moves, since the $O Z$ axis is taken to be in the instantaneous direction of camera motion, the co-ordinate $Z$ changes while $X$ and $Y$ remain constant. The OMA $\alpha$ may also change. We assume that other rotational components of the camera motion are small enough to be neglected. Hence for small changes $\Delta Z$ and $\Delta \alpha$ in $Z$ and $\alpha$, the corresponding changes $\Delta x$ and $\Delta y$ in the co-ordinates on the image position of the point $\mathrm{P}(X, Y, Z)$ can be approximated by

$$
\left\{\begin{array}{c}
\Delta x=-\frac{\Delta Z}{Z f}(x \cos \alpha-f \sin \alpha)(f \cos \alpha+x \sin \alpha)+\frac{x^{2}+f^{2}}{f} \Delta \alpha \\
\Delta y=-\frac{\Delta Z}{Z f} y \cos \alpha(f \cos \alpha+x \sin \alpha)+\frac{x y}{f} \Delta \alpha
\end{array}\right.
$$

We refer to $\Delta Z, \Delta \alpha$ and $\alpha$ as the motion parameters. The co-ordinates, $X, Y$ and $Z$ are uniquely determined by the image position $(x, y)$, the image difference $(\Delta x, \Delta y)$, the focal length $f$, and the motion parameters. The image difference can be measured from two consecutive images after correspondences have been matched. However, because it is not possible to control the camera motion in the sewer, the motion parameters cannot be obtained directly. The following technique was developed to obtain the motion parameters by studying the image changes over the whole image plane.

During motion, image difference will be governed by the equations (2). Image difference as a function of position on the image plane comprises the image flow field. Obvions:y, 
the image flow field is determined by the relative motion between the camera and the pipe. Eliminating $\mathrm{Z} / \Delta \mathrm{Z}$ from equations (2), gives

$$
x y \Delta \alpha \tan \alpha+y f \Delta \alpha-f \Delta y \tan \alpha=y \Delta x-x \Delta y
$$

Equation (3) illustrates the relationship between the image flow and motion parameters $\Delta \alpha$ and $\alpha$. By analysing a pair of consecutive images, the image flow field can be obtained. This means that in the image pair under investigation, the image difference, $\Delta$ $x$ and $\Delta y$, at position $(x, y)$ is known. The variables needed from equation (3) are $\alpha, \Delta \alpha$ and $f$. From any three pairs of corresponding points, an estimate of the motion parameters can be obtained. To improve accuracy, the least squares method was used.

An accurate estimate of the position of the spatial point $\mathrm{P}$ can be made, associated with the estimation of $\Delta Z$, in the following manner. It is assumed that during the period when the camera took images 1,2 and 3 (Fig.1), the camera travelled in a straight line at constant speed. So, $\Delta Z_{1}=\Delta Z_{2}=\Delta Z$. The position of a given $P$ can be measured as $\left(X_{1}\right.$, $\left.Y_{1}, Z_{1}\right)$ from images 1 and 2 , and as $\left(X_{2}, Y_{2}, Z_{2}\right)$ from images 2 and 3 . These two measurements should have the following relationship

$$
X_{1}=X_{2}, \quad Y_{1}=Y_{2}, \quad Z_{1}=Z_{2}-\Delta Z
$$

It is now possible to obtain an estimate of $\Delta Z$ and $P$.

\section{Evaluation of the Error}

The deformation of a sewer may be only very small before its collapse, so high spatial resolution is required. In analysing the applicability of a proposed approach, it must be known how susceptible to error that approach is.

All computer vision techniques involve two procedures: a large scene is mapped onto a plane of small dimensions by a camera (the imaging procedure), and this plane is then digitised and input into a computer (the digitisation procedure). The limited resolution of the imaging system (which may be interpreted as the number of sensory elements in the CCD array used in the camera or the size of image buffer on the image grabbing board) requires the whole scene to be mapped on an $m$ by $n$ array of points, for some fixed $m$ and $n$. This array may not be large enough to represent scene adequately. As a result a significant amount of quantisation error is introduced into computations that involve the location of image points and features. Note that this type of error cannot be reduced by implementing the approach more accurately and carefully. Rather it is caused by the inherent limitations of the instruments used[5]. The co-ordinate most prone to error is the depth, $Z$, which is given by

$$
Z=-\Delta Z \frac{\left(x_{1} \cos \alpha-f \sin \alpha\right)\left(f \cos \alpha+x_{1} \sin \alpha\right)}{f\left(x_{2}-x_{1}\right)-\left(x_{1}^{2}+f^{2}\right) \Delta \alpha}
$$

where $\left(x_{1}, y_{1}\right)$ and $\left(x_{2}, y_{2}\right)$ are a pair of corresponding points on image 1 and 2 . 
We assume that $\Delta Z, \alpha, f$ and $\Delta \alpha$ have been calculated accurately as described in the previous section. Then the depth error for each individual point depends only on the errors in locating the $x$ co-ordinates of the two corresponding points. If there are errors, denoted by $\varepsilon x_{1}$ and $\varepsilon x_{2}$, in locating a pair of corresponding image features, the relative error, $\varepsilon Z / Z$, in the depth estimate of the spatial point can be approximated by $\varepsilon Z / Z=\Gamma_{1} \varepsilon x_{1}+\Gamma_{2} \varepsilon x_{2}$, where $\Gamma_{1}$ and $\Gamma_{2}$ can be calculated in term of image position, image difference and motion parameters.

To evaluate how error-prone a given approach is, a realistic measure of the error should be given. One measure is the maximum error that can occur in applying the approach on the assumption that $x_{1}$ and $x_{2}$, have been measured to the nearest pixel. In general, however, knowledge of the maximum error is not very important, as the actual error is often far less than the maximum. A better measure in practice is the average error which is the mean of the absolute values of the error over all possible value of the quantisation errors. A plot of the average error distribution with respect to $X$ and $Z$, is given in Fig.3. In the cases most commonly used the average error is less then $0.4 \%$ of the measured distance, $Z$.

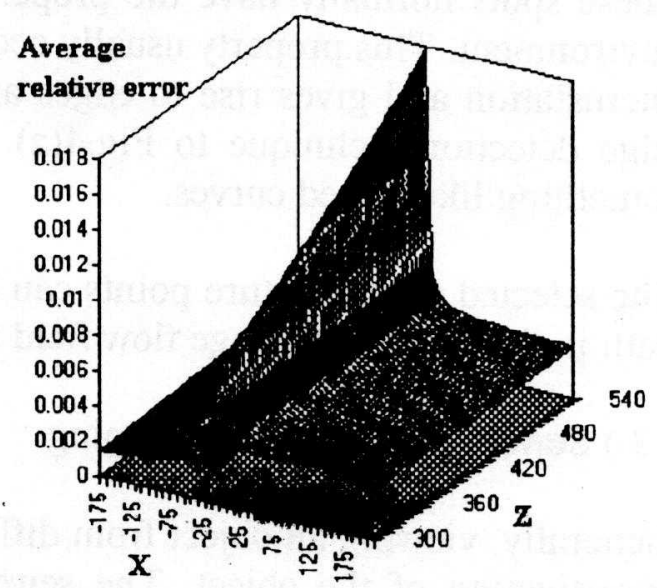

Fig. 3 The average error distribution The diagram illustrates that the further away from the tine camera motion a point is (the bigger the value of $\mathrm{X}$ ), the more accurate is the measurement produced by the proposed approach.

\section{Implementation}

Based on the proposed approach, a combined system has been built. This system consists of three major components, (1) Image sequence grabbing, (2) Image sequence analysis, (3) Sewer structure data mapping.

\section{( 1 ) Image Sequence Grabbing}

The proposed approach requires that the gap between two viewpoints be very small in order to obtain an accurate instantaneous image flow field. For a documentary video tape recorded by a moving camera, the smallest possible gap is the displacement between the two viewpoints where two consecutive images were taken. A normal video tape runs at a rate of 25 frames per second. The equipment required to acquire every frame consecutively without the loss of data associated with image compression is complicated and quite expensive. Considering the balance between the price of instruments and the accuracy required from the engineering view of point, the proposed system grabs image sequences at a rate of every other frame of a normal running video. Fig. 4 shows a pair of sewer images. Image (b) was taken after the camera had moved one "step" forward after image (a) was taken. 


\section{( 2 ) Image Sequence processing and analysis}

Using a cross-correlation technique, the image flow field can be detected. Fig. 5 shows the image flow field between the images in Fig.4. The camera motion parameters can be obtained by the procedure described above.

In the sewer survey application, the sewer structure is determined by the estimation of the $3 \mathrm{D}$ position of a set of feature points lying on the pipe wall. In a captured image, any spot can be used as a feature point providing it can be identified in consecutive frames. These spots normally have the property that their light intensity is distinct from their environment. This property usually occurs at the images of junctions, cracks, and surface encrustation and gives rise to edges and corners. Fig. 6 shows the result of applying an edge detection technique to Fig.4(a). It may be noticed that the junctions appear as something like closed curves.

The selected image feature points can be matched from one image to the next, along the path indicated by the image flow field and their 3D position recovered.

\section{( 3 ) Sewer Structure Data Mapping}

Generally, viewing an object from different angles can be helpful in facilitating a proper investigation of the object. The sewer structure data obtained is managed through a Graphical User Interface (GUI) which allows the user to view the shape of the sewer from different viewpoints. Fig.7 demonstrates some examples of viewing two sewer junctions from two different view points. A cube model is used to illustrate the relative position between the user and the object of observation.

\section{Conclusion}

Sewer survey is very important in maintaining a sewer system in good working condition. This long term, data accumulation task produces a large quantity of data to be analysed. Obviously, computer vision techniques show great promise for automatic analysis of those data. This paper presented a Sewer Survey System which demonstrates the feasibility of the proposed computer vision technique.

\section{Acknowledgement}

The authors would like to thank the Science and Engineering Research Council (SERC) for funding this project, and all their colleagues in the Artificial Intelligence Laboratory at Sheffield Hallam University for their active participation. 


\section{References}

[1] S. J. Broadhurst, T. P. Pridmore and N. Taylor. Sensing for Feature Identification in Sewers. Automation and Robotics in Construction XI (Ed. D. A. Chamberlain) Proceedings of the 11th International Symposium on Automation and Robotics in Construction( ISARC) Brighton, UK, 24-26 May, 1994. ISBN : 0444820442

[2] E. D. Micheli, V. Torre and S.Uras. The Accuracy of the Computation of Optical Flow and of the Recovery of Motion Parameters. IEEE Trans. on Pattern Analysis and Machine Intelligence Vol. PAMI-15 No. 5 May 1993

[3] Allen M.Waxman and Shimon Ullman. Surface Structure and Three-Dimensional Motion from Image Flow Kinematics. The International Journal of Robotics Research Vol 4, No. 3 Fall 1985

[4] Roger Y.Tsai and Thomas S. Huang. Uniqueness and Estimation of ThreeDimensional Motion Parameters of Rigid Object with Curved Surface. IEEE Trans. on Pattern Analysis and Machine Intelligence Vol. PAMI-6 No. 1 January 1984

[5] Behrooz Kamar-Parsi and Behzad Kamar-Parsi. Evaluation of Quantization Error in Computer Vision. Computer Vision and Pattern Recognition, June 1988 pp 52-60 


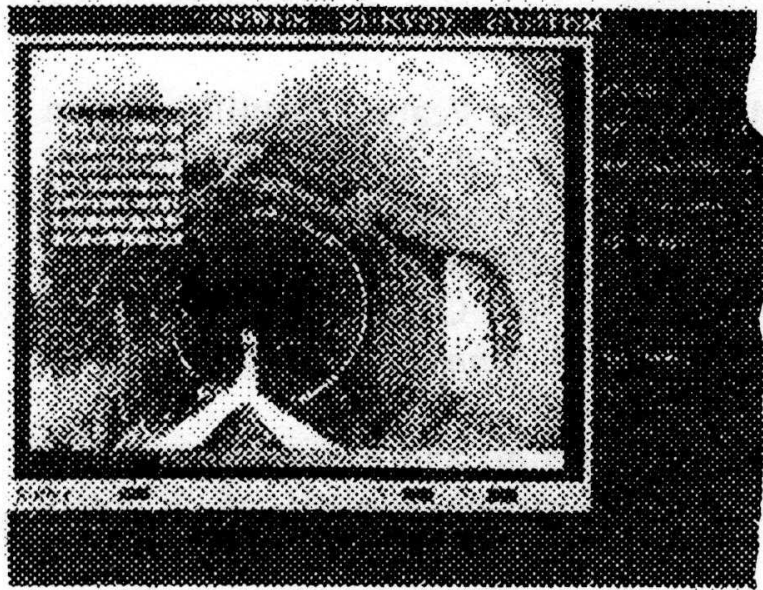

(a)

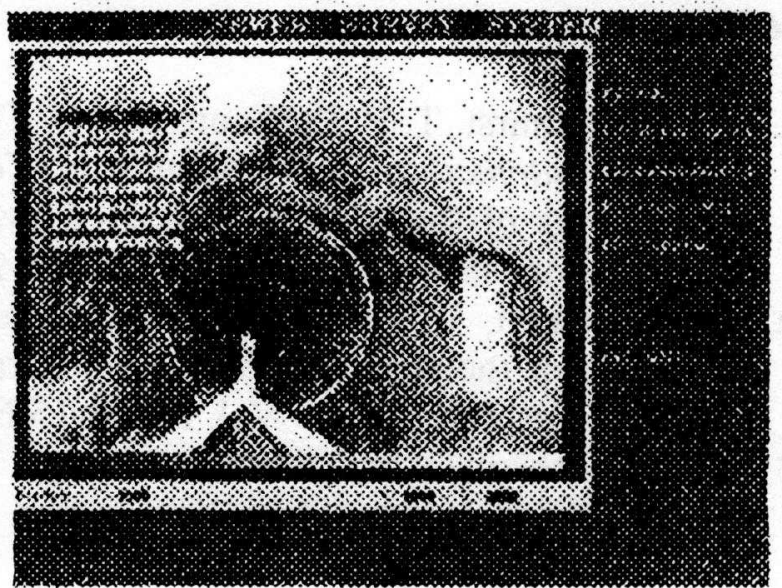

(3)

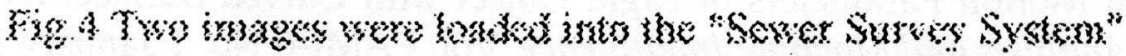

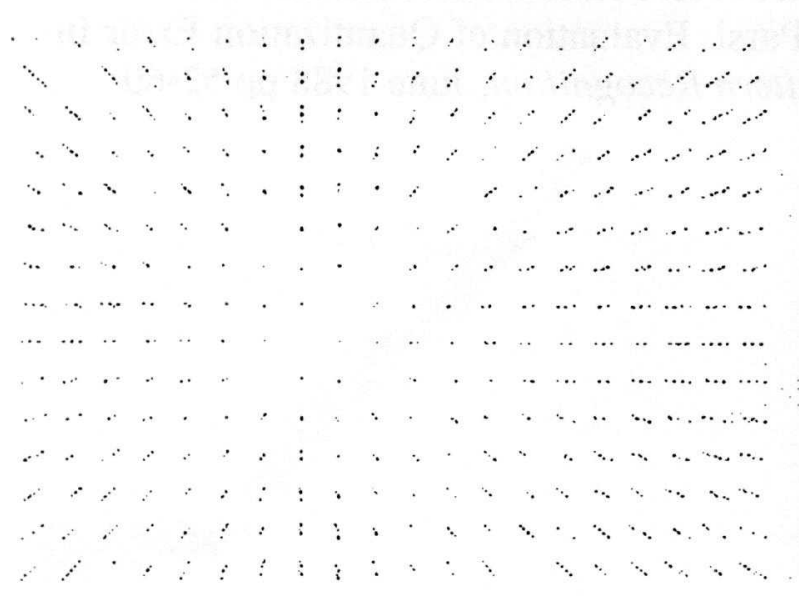

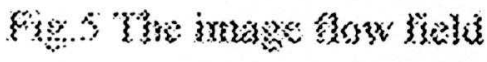

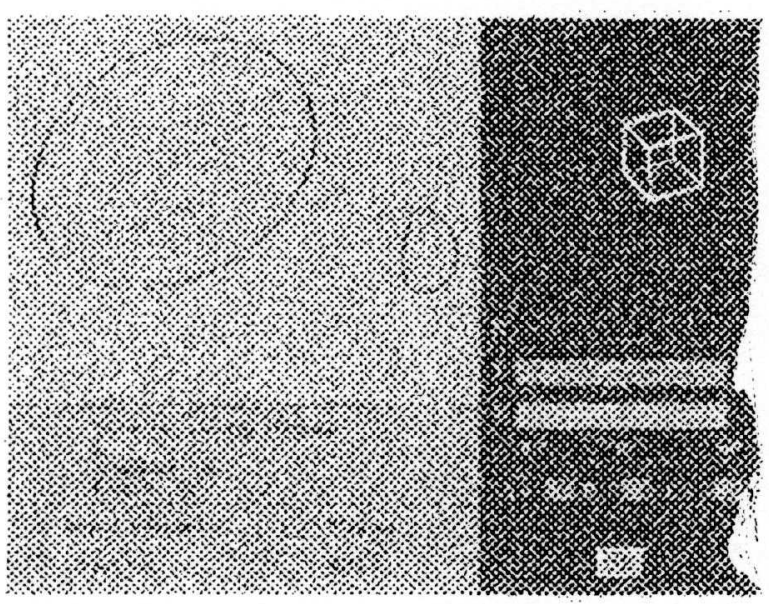

(a)

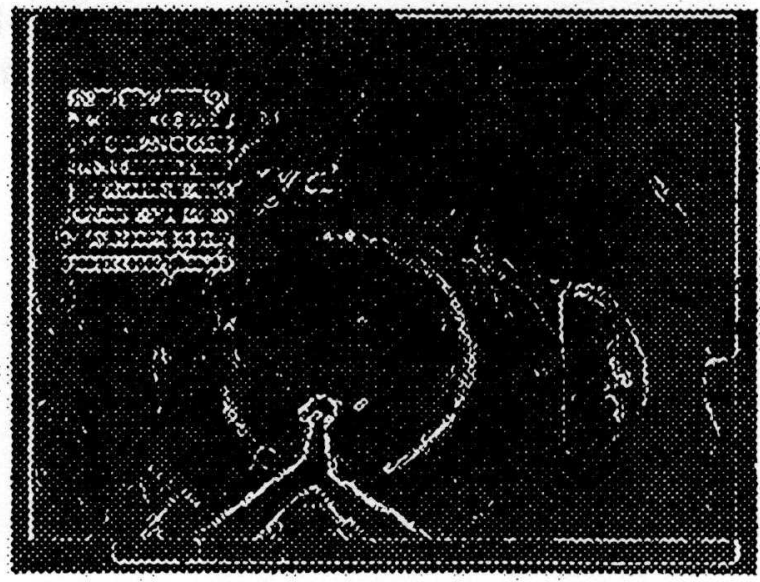

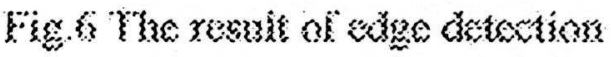

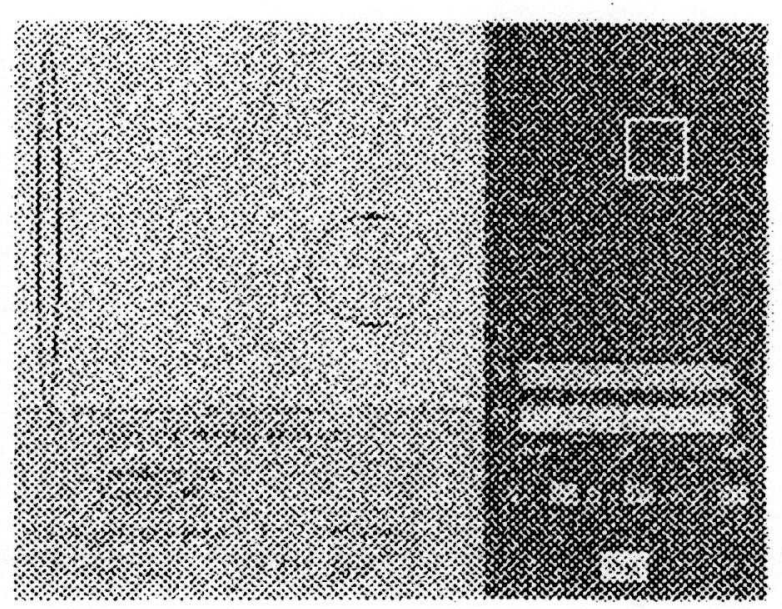

(i)

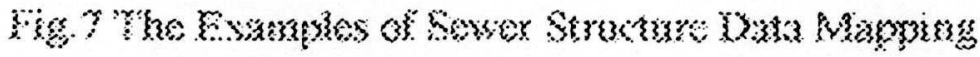

